\title{
The significance of geographic location in island studies: a rejoinder
}

\author{
Harvey W. Armstrong \\ University of Sheffield \\ $U K$ \\ h.armstrong@sheffield.ac.uk
}

and

Robert Read

University of Lancaster

$U K$

r.read@lancaster.ac.uk

\begin{abstract}
This rejoinder seeks to build upon McElroy and Lucas' excellent paper by exploring further the relationship between distances from small islands to their main markets and economic performance. It is argued that the nature of this relationship is not only statistically very strong but also that it is nuanced and multifaceted. A very fruitful set of areas for further future research awaits any researcher brave and energetic enough to pick up the baton and run (or swim - we are talking islands here) with it.
\end{abstract}

Keywords: distance decay, great circle distance, economic potential, intervening opportunities theory, islands, niche markets

(C) 2014 - Institute of Island Studies, University of Prince Edward Island, Canada.

\section{Introduction}

The new contribution by McElroy and Lucas to the debate on the role of 'geography' in the performance of small island economies is a very welcome one. The authors have unerringly put their finger on what may actually be the key determinant of differential economic performance among small islands across the global economy: geographical accessibility. If it is not the key determinant, and this is something which only future research can assess, then it must surely be eventually shown to be one of the two or three principal influences on economic performance. The paper is yet another in a long line of outstanding contributions by Jerry McElroy and colleagues to the systematic analysis of small island economies, particularly of course, small island tourism economies (SITEs), and it is to be welcomed as such.

\section{Great circle distance}

We ourselves have, since 2006, regularly used the particular measure of accessibility used in the McElroy and Lucas paper (great circle distance to three key global regional markets GCD) in our own research on differences in the economic performance of small states (e.g. Armstrong \& Read, 2006). The variable invariably does well in the sense of 'explaining' a statistically significant part of the variance in economic performance. In this sense, our results 


\section{H. W. Armstrong \& R. Read}

are in close accordance with the new McElroy and Lucas findings. In this rejoinder we will focus solely on the accessibility variable, on what it it may or may not be showing us and, above all, what steps might be possible in future research to refine the analysis of accessibility for small island economies.

When we ourselves began to first incorporate geographical distance variables in our statistical analysis of the economic performance of small states and island economies, we adopted the rather crude approach of experimenting with different specifications based on great circle distances from each small state to the wider global economy, and then simply adopted the one which produced the greatest amount of statistical 'explanation'. The result was the variable used by McElroy and Lucas, namely great circle distance from the capital of the island (in our research, of the small state) to the nearest of three major global regional markets: Brussels (for the European Union), whichever is the nearer of Washington DC or Los Angeles (for North America), and Tokyo (for East Asia). As McElroy and Lucas show, this variable has been remarkably durable and successful, in a statistical sense, in accounting for variations in national income per capita. We ourselves prefer to use the logarithm of GCD rather than GCD itself, for two reasons. Firstly, in the kinds of models we have used (e.g., censored regression, OLS) the variables need to be linearized and log-linearization turns out to be the best way of achieving this. Secondly, we would argue that there are sound economic reasons for expressing the relationship between geographical distance and economic performance as a non-linear one. For a start, transport costs (both marine and air transport modes) are non-linear with geographical distance, partly because of the importance of fixed costs relative to line-haul costs and partly because of the famous 'taper principle' in line-haul costs. This non-linearity applies not only to transport money costs but also to the important time costs. The direct link between the cost of overcoming remoteness and economic performance means that the relationship between the two can also be expected to be non-linear.

In addition to the transport costs argument for a non-linear relationship between remoteness and economic performance, the theory and analysis of agglomeration economies also suggests a strongly non-linear relationship. Economic interactions across geographical space often display a strongly exponential (or other non-linear functional form) pattern of 'distance decay', ranging from commuting trips and migration to trade in services and manufactured products. The non-linearity of 'distance decay' functions has long been recognized in economic geography as having almost the status of a 'law', and it is reassuring to discover that small states and islands do indeed seem to conform to this same 'law'. The sort of gain in 'explanation' one obtains by linearizing the function can be illustrated if one correlates GNI per capita against GCD. Using 2010 World Bank data for GNI per capita and GCD for 73 island states and associated territories one obtains a correlation coefficient of -0.456 for a linear relationship and a coefficient of -0.490 if the logarithm of GCD is used rather than simple GCD. This may not seem a huge gain, but our own research shows that the gain is even higher when the variable is used as part of a wider multivariate model rather than a simple bivariate correlation.

Let us return to the GCD variable (linearized or otherwise!) and attempt to 'unbundle' it as a variable in order to try to see what it is actually saying to us. As noted earlier, when we first adopted the variable, we based its choice mainly on the rather lazy and almost tautological principle of it being selected because it gave us the best results! We did, however, experiment with a series of different specifications for the variable and, although we never published the results of these, the findings are quite interesting. Returning to these experiments, but this time 
using 2010 GNI per capita data for 73 global island states and associated territories, one obtains the following correlation coefficients between GNI per capita and GCD (see Table 1):

Table 1: Correlation coefficients between GNI per capita and GCD.

1. GCDEU: great circle distance from the island to solely the EU (log, $\mathrm{km}) \quad-0.445$

2. GCDGLOB3: GCD to nearest of EU, USA or E. Asia (log, $\mathrm{km}) \quad-0.490$

3. GCDREG: GCD to main city in island's global region (World Bank definition of region) (log, km) $\quad-0.390$

4. GCDNEAREST: GCD to nearest large city (e.g. Auckland for many South Pacific islands) $(\log , \mathrm{km})$

5. GCDMETROP: GCD to capital city of former or existing metropolitan power (e.g., London for Cayman Islands, Paris for Tahiti) (log, $\mathrm{km}$ ) $-0.395$

\section{Regionalization, not globalization}

What is particularly interesting about these results is that they suggest that other specifications of the 'distance decay' variable can also produce statistically significant results. One cannot simply plug them all (or indeed any others one might think of) into econometric analysis because they are highly multicollinear variables. A choice among them has to be made. As noted earlier, we elected to use the one with the highest level of explanation. To be fair to ourselves, there was, in fact, a second reason for selecting GCDGLOB3 which has more logic to it. In our earlier pre-2006 research, we had gradually come to the conclusion that, as far as small states are concerned, the much-vaunted globalization view was not being borne out. Most of the small states we examined seemed to be very heavily dependent on their own global 'region'. In other words, the world of small states seemed to us to be more one of 'regionalization' than 'globalization'. As a result, most small states seemed to us to be filling export niches for their nearby region rather than the full global economy. Since, at the time of the 2006 research, the three dominant global regions were East Asia, North America and Europe, it was a short step to the GCD variable used by ourselves and in the new McElroy and Lucas note. We would defend the continuing preference for measures such as GCDGLOB3.

An interesting question for the future is whether GCDGLOB3 is the best specification for a remoteness variable. Even in 2006 it was clear to us that more ideal specifications were possible. International trade analyses and sub-national (and sub-EU) regional analyses almost invariably try to use gravity-model based specifications for geographical distances between local economies and their wider markets. At their very best, these measures are very good indeed and modern GIS methods are greatly facilitating this type of research (e.g. Gutierrez, Condeço-Melhorado \& Martín, 2010). 'Economic potential' is usually measured as a combination of distances (or costs or time) from one economy to all of the others in the set. The measures are non-linear (to allow for 'distance decay') and the economies at either end of each link are usually weighted by size (typically population, workforce or GDP weights are applied to allow for the fact that a link to a big trading partner is more important than that to a small partner).

Unfortunately for research on small islands, it is almost impossible, even now, to produce similar measures for small island economies. There are two reasons for this. Firstly, 


\section{H. W. Armstrong \& R. Read}

one would need good weighting data (e.g. GNI) for every single economy, large and small, in the whole world! Since there are gaps in such data, and it is the smallest states which tend to be most commonly omitted, the task remains an impossible one. Secondly, small island economies are, by definition, islands. Gravity model measures of remoteness are very good for on-land transport connections but it has proved almost impossible to combine on-land links with sea-transport links in any meaningful manner. Hence, in the absence of a sensible and comprehensive gravity model specification, we were forced back on the rather cruder GCD specification.

Rolling forward to the present day from 2006, a further problem with our GCDGLOB3 variable can easily be seen. The emergence of the BRIC economies and, in particular, of China and India, has rapidly begun to shift the economic centre of gravity of the global economy. The global economy is now multi-centred and our original threefold specification of East Asia, North America and Western Europe is now out of date. Or is it? Perhaps the change as far as small island economies has not been as radical as for the bigger global states because of their regional rather than global disposition. This is surely an issue of importance for future research on island economies. At the very least, there is a case for experimenting with more up-to-date specifications of GCD which allow for the newer centres of economic power.

\section{Avenues for future research}

Let us assume that remoteness measures, such as GCD, continue to be important for understanding why small island economies differ so greatly in their economic performance. If one is willing to make this assumption, then other avenues of potentially fruitful future research spring to mind. Three in particular have long exercised us, but sloth and other interests have sadly diverted us from these!

The first of these is the nature of the export niches (e.g., different types of tourism, financial services, manufacturing niches) on which so many small island economies depend. An interesting feature of the economic performance of small island states is that many of the poorer performing economies are to be found in regions of the world which have large clusters of small island states (notably the Pacific economies, but also in the Caribbean and subSaharan Africa). This raises the intriguing possibility that small islands in such clusters are competing for a finite (probably very limited) number of niches. For example, just how many cruise tourism island economies can the Caribbean sustain? Or how many offshore financial centres can Europe sustain? Interestingly, economic geographers have long had precisely the kind of theory necessary to analyse this type of situation: intervening opportunities theory. What this argues is that, even within a gravity model view of the effect of distance decay, a further influence is whether between one's own island and the main market there is one or more 'intervening opportunity' islands. In the presence of distance decay, the theory argues, tourists and customers of products and the like will tend to select the nearest intervening opportunity (other things being equal). One then has to ask how the intervening opportunity case managed to get started in the first place. Two plausible hypotheses are: (a) that the process is a random one; and/or (b) one island got there first - the 'first mover' hypothesis - and once established became hard to displace! To our knowledge, no one has yet attempted an intervening opportunities model analysis of small island clusters. It would be most interesting to see this attempted. The implication is that distance decay (i.e., GCD) is not enough on its own. The distance decay needs to be moderated by an intervening opportunities variable (either 
in time or space, or both). Since the numbers of niches to be filled are relatively limited, it is inevitable that small islands in large clusters are more likely to find themselves the losers and, hence, more poorly performing islands will inevitably be disproportionately found in such clusters. This is exactly what we observe globally.

The second distance decay-related issue which has long exercised us but which we have not got around to addressing is the possibility of sectoral variation in the distance decay process. For the sake of argument, let us stick with McElroy's small island tourism economies (SITEs). In these islands, a very pertinent question is whether the distance decay process is the same for each of the main tourism niches. Four obvious tourism niches are: cruise tourism (particularly interesting because of its very rapid recent growth); overnight-stay tourism (particularly 'sun and sand' longer stay tourism); day-trip tourism; and activity tourism (including 'cold water' activity tourism). There are, of course, many other sub-categories of tourism but, for the sake of developing the argument, let us focus on just these four. One can see immediately that the distance-decay process for day-trip tourism must be remarkably different to those for cruise, overnight and activity tourism. The coefficient on the distance decay function for day-trip tourism must surely be much larger than for the other two and, in some cases, the function may well be simply truncated at some critical distance. Interestingly, only the Bahamas in the Caribbean region has large numbers of day-trippers; surely because of the large size of the distance decay coefficient and the proximity of South Florida. There must also surely be big differences in the distance decay coefficients between overnight-stay, activity and cruise tourism although it is unclear precisely what these are since so little research has been done on the nature of their different distance decay functions from the perspective of small islands. One suspects that the distance decay function coefficients will be higher for overnight-stay tourists than those for cruise passengers or activity tourists but we simply do not know this for island economies. One can make similar arguments for manufacturing trade but financial services are much less distance-determined. There is a fruitful area of research here for anyone with the energy and enthusiasm to pursue it.

The third issue which has long exercised us is whether, in some cases, other variables can over-ride or negate the distance-decay 'law' of economic behaviour. In some recent research on Caribbean small states, we were intrigued to discover that, for some of the associated territories in the region, tourist flows from far-distant Europe actually exceed those from the huge nearby North American market. Are there language and culture variables at work here over-riding distance decay? Or, could it be that air transport links and infrastructure development have favoured flights from, say, Amsterdam to the Netherlands Antilles or from London to, say, the Cayman Islands rather than direct links to the USA? As well as being an interesting topic in its own right, this possibility shows us that one must never be too deterministic in applying distance decay variables to island states. Other variables do matter and, hence, it is vital to incorporate distance variables within a properly and fully specified multivariate model, as McElroy and Lucas have done.

\section{References}


Armstrong, H.W., \& Read, R. (2006). Comparing the economic performance of different microstates, and between microstates and larger countries. World Development, 26(4), 639656.

Gutierrez, J., Condeço-Melhorado, A., \& Martín, J.C. (2010). Using accessibility indicators and GIS to access spillovers of transport infrastructure investment. Journal of Transport Geography, 18(1), 141-152. 